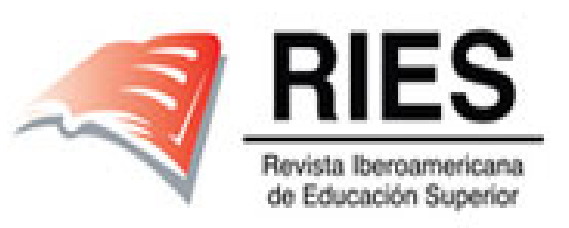

Revista Iberoamericana de Educación Superior

E-ISSN: 2007-2872

emmaro@unam.mx

Instituto de Investigaciones sobre la

Universidad y la Educación

México

de Vries, Wietse; Vázquez-Cabrera, Rebeca; Rios-Treto, David

Millonarios o malparados: ¿de qué depende el éxito de los egresados universitarios?

Revista Iberoamericana de Educación Superior, vol. IV, núm. 9, octubre-diciembre, 2013, pp. 3-20

Instituto de Investigaciones sobre la Universidad y la Educación

.jpg, México

Disponible en: http://www.redalyc.org/articulo.oa?id=299126789001

Cómo citar el artículo

- Número completo

- Más información del artículo

Página de la revista en redalyc.org

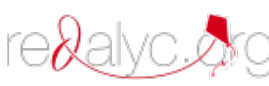

Sistema de Información Científica

Red de Revistas Científicas de América Latina, el Caribe, España y Portugal

Proyecto académico sin fines de lucro, desarrollado bajo la iniciativa de acceso abierto 


\title{
Millonarios o malparados: ¿de qué depende el éxito de los egresados universitarios?
}

\author{
Wietse de Vries, Rebeca Vázquez-Cabrera y David Rios-Treto
}

\begin{abstract}
Resumen
La literatura sobre la educación superior y el mercado de trabajo suele señalar que entre más alto el nivel educativo, mayor éxito en el mercado laboral en términos del salario percibido. Sin embargo, en un estudio sobre egresados universitarios en México encontramos diferencias abismales entre personas con el mismo nivel de preparación. Si bien para la población en general se podrían explicar las diferencias en ingresos por la educación recibida, en el caso de egresados universitarios este factor parece poco plausible. En este artículo, a partir de datos de egresados de nueve universidades mexicanas, revisamos cuáles otros factores podrían explicar las diferencias salariales entre personas con el mismo nivel educativo.
\end{abstract}

Palabras clave: egresados, mercado laboral, salarios, preparación, competencias.

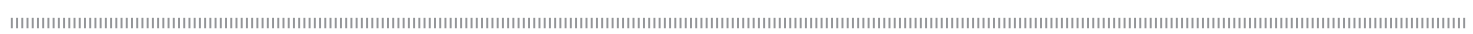

Milionários ou malparados: do que depende o êxito dos formados universitários?

\section{Resumo}

Os estudos sobre educação superior e mercado de trabalho normalmente colocam que, em termos de salário percebido, a maior nível educativo corresponde maior êxito no mercado de trabalho. Entretanto, em uma pesquisa sobre formados universitários no México achamos diferenças abismais entre pessoas com o mesmo nível de preparação. Embora para a população geral estas diferenças no nível de renda poderiam ser explicadas pela educação recebida, no caso dos formados universitários este fator parece pouco provável. Neste artigo, a partir de dados de formados de nove universidades mexicanas, revisamos que outros fatores poderiam explicar as diferenças salariais entre pessoas com o mesmo nível educativo.

Palavras chave: formados, mercado de trabalho, salários, preparação, competências.

Wietse de Vries

Doctor en Educación por la Universidad Autónoma de Aguascalientes, México. Profesor-investigador de tiempo completo en la Benemérita Universidad Autónoma de Puebla, México. Miembro del Sistema Nacional de Investigadores, nivel II. Temas de investigación: políticas públicas en educación superior, evaluación, financiamiento, efectos en actores (instituciones, académicos, estudiantes, egresados).

Rebeca Vázquez-Cabrera

beka.vazkez.cabrera@live.com.mx Egresada de la licenciatura en Educación por la Universidad Autónoma Juárez de Tabasco, México. Participante en el Verano Científico en 2012 con apoyo de la Academia Mexicana de Ciencias.

\section{David Rios-Treto}

Egresado de la licenciatura en Educación por la Universidad Autónoma de Tamaulipas, México. Participante en el Verano Científico en 2012, con apoyo de la Academia Mexicana de Ciencias. Actualmente labora en la Universidad Autónoma de Tabasco, México. 


\section{Abstract}

The literature on higher education and the job market usually points out that the higher the educational level, the greater the success in the labor market in terms of received income. However, in a study on university graduates in Mexico we found enormous differences between individuals with the same level of training. Although for the general population the differences in income could be explained by their educational level, with regard to university graduates this factor does not seem to be plausible. Based on data about graduates from nine Mexican universities, this article reviews which other factors might explain the differences in the income of individuals with the same educational level.

Key words: graduates, job market, income, training, competencies. 


\author{
Yeah, they say you can make it if you try \\ Yet, some of this generation is millionaires \\ It's hard for me to keep decent clothes to wear \\ Otis Rush, Double Trouble
}

\section{Introducción}

En un estudio de seguimiento de egresados en el que participaron nueve universidades mexicanas (seis públicas y tres privadas) encontramos diferencias salariales grandes dentro de una población nominalmente uniforme: en todos los casos se trata de egresados que habían terminado exitosamente su carrera en el mismo periodo y que fueron entrevistados sobre su situación laboral en el mismo momento, a cuatro o cinco años de haber egresado. Todos contaban con un mismo nivel educativo, expresado en un título de licenciatura formalmente reconocido. ¿Qué, entonces, podría explicar las diferencias en salarios percibidos?

\section{El éxito visto desde el mercado laboral}

Definir el éxito del egresado a partir del ingreso mensual percibido obviamente tiene sus complicaciones. Una primera dificultad consiste en que el salario - $-\mathrm{y}$ su distribución - puede depender de la situación económica del país, de la región o del comportamiento de un sector económico específico. Así, el ingreso reportado puede variar de manera importante según el momento de la entrevista.

Un segundo problema, como observan Cabrera et al. (2003), es que el sueldo refleja solamente un aspecto de la vida laboral. Un salario más alto no equivale automáticamente a mayor satisfacción con el trabajo, ni a una buena coincidencia entre estudios y empleo, ni a una mayor satisfacción con la universidad o la formación recibida.

Sin embargo, considerar estos factores crea problemas adicionales. La satisfacción que reporta el egresado con su empleo o con la universidad puede ser un indicador muy subjetivo: el egresado puede basar su opinión en sus esperanzas laborales iniciales, en una comparación con sus padres o con sus colegas, o en el salario que percibe. Es decir, la valoración que hace el egresado de la satisfacción con su trabajo puede depender de una multitud de factores y, a su vez, la valoración retrospectiva de su experiencia universitaria puede estar condicionada por su situación laboral y personal en el momento de la entrevista (Pike, 1994).

Un tercer problema radica en inferir que el ingreso promedio de los egresados refleja la calidad de una institución de educación superior. Generalmente no es claro qué aspectos de la experiencia universitaria contribuyeron al éxito o al fracaso.

Así, la mayoría de los estudios sobre egresados universitarios y el mercado laboral suele considerar al ingreso como un dato clave para medir el éxito. El egresado exitoso es aquel que tiene un buen sueldo, estatus, poder e influencia social (Teichler, 2003). A partir de esta definición se han efectuado varios tipos de seguimiento de egresados.

Un primer tipo de estudios se basa en datos como las encuestas nacionales sobre la población económicamente activa (PEA) y sus características. Estos estudios suelen confirmar que conviene estudiar una carrera universitaria, ya que los titulados reciben ingresos más altos en comparación con la población que no accedió a la educación superior. Así, continúan corroborando teorías como la del capital humano: entre más años de escolaridad, mayores ingresos (Becker, 1964).

Al mismo tiempo, estos estudios suelen señalar que hay ciertos desajustes entre la educación superior y el mercado laboral. En algunas áreas, los egresados parecen carecer de la preparación que el mercado demanda, mientras que en otras están sobrecalificados (Sicherman, 1991). 
Sin embargo, estos estudios sobre la PEA tienen sus limitaciones, ya que brindan poca información acerca de factores que podrían explicar las diferencias salariales, tales como la preparación recibida, el tipo de institución, el tipo de carrera, o las condiciones familiares o educativas del joven anteriores a su ingreso a la universidad (Teichler, 2003). Es decir, en estos estudios la educación figura como ceteris paribus: se presupone que todos los que recibieron 10 años de educación pasaron por experiencias muy similares y que cuentan con el mismo nivel educativo. Así, no logran responder a la pregunta que nos preocupa aquí: ¿porqué hay diferencias salariales abismales entre egresados universitarios?

Un segundo conjunto de estudios se refiere a los estudios de egresados. En un contexto en que crecientemente se pide a la universidad rendir cuentas, estos estudios son cada vez más comunes. Sin embargo, dado que los grupos externos de interés prefieren ver evidencias sobre la contribución de la universidad al desarrollo nacional o regional, también estos estudios suelen concentrarse en los resultados socioeconómicos que acarrea la formación universitaria. Como señalan Cabrera et al. (2003), a partir de una revisión de la literatura estadounidense, el 70\%de los estudios enfatiza el aspecto socioeconómico, mientras que sólo el quince $\%$ revisó el aprendizaje y otro quince $\%$ documentó las contribuciones financieras que hacen los egresados a su alma mater.

Estos estudios también tienen fuertes limitaciones: una primera es que cada universidad —en ocasiones cada carrera- suele hacer su propio estudio con su particular metodología, y divulgar sólo la información que más le conviene. Con ello, las comparaciones entre universidades se vuelven imposibles. Una segunda limitación es que estos seguimientos raras veces incluyen o analizan factores institucionales o individuales que podrían explicar a qué se deben las diferencias de ingresos de los egresados en el mercado laboral.
Así, un factor en común de esta diversidad de estudios es que se concentran en la inserción en el mercado y en el ingreso que percibe el titulado, pero no analizan la aportación que hizo la educación superior.

\section{La visión desde la educación}

En contraste con los estudios socioeconómicos de egresados y mercados de trabajo, la literatura sobre el funcionamiento de los sistemas educativos suele indicar que muchas desigualdades sociales se producen o reproducen dentro del sistema, indicando una amplia gama de posibles factores. Durante los sesenta del siglo pasado, nació la tesis de que el origen socioeconómico condicionaba el acceso a la educación y las posibilidades de perdurar en ella. De este modo, la educación básicamente pareciera reproducir o ratificar las desigualdades sociales existentes (Boudon, 1983; Bourdieu y Passeron, 1977, 2003).

Esta tesis dio lugar a varias políticas con el objetivo de democratizar la educación y procurar mayor equidad (Bolívar, 2005). Estas reformas incluyen acciones para ampliar el acceso, la introducción de un currículo uniforme nacional, o la discriminación positiva de grupos menos favorecidos, con la idea de que una educación similar para todos aminoraría las desigualdades sociales y económicas en el futuro.

Sin embargo, tales reformas no parecen ser la panacea para la desigualdad (Bowles y Gintis, 2000, 2002; Scott, 2005). Según algunos autores, cierta desigualdad es ineludible, ya que la educación selecciona por méritos, y diferentes grupos sociales tienen distintas experiencias (Arrow et al., 2000). Las reformas educativas pueden atender la inequidad por el lado del acceso a la educación, para reducir la influencia de factores como la herencia educativa familiar (la reproducción), pero eso no garantiza que desaparezcan las desigualdades en el mercado laboral (la producción) (Dubet 
y Martuccelli, 1998). Así, promover la igualdad de acceso a oportunidades educativas no resuelve automáticamente la desigualdad en resultados, tanto dentro del sistema educativo como en el mercado laboral (World Bank, 2006, 2008; IDB, 2008), ya que los estudiantes tienen diferentes capacidades de aprendizaje, amén de distintas circunstancias, credenciales, intereses y expectativas frente al mercado de trabajo (Roemer, 1998; Collins, 1979).

La igualdad de oportunidades de acceso, permanencia y titulación se vuelve más complicada en la educación superior, donde existe una gran variedad de tipos de instituciones, carreras, currículos y estudiantes. Eso ocasiona que la vinculación entre mercado de trabajo y universidad parece estar mediada por una enorme diversidad de factores. Parte de esta complejidad se debe a los cambios en la educación superior misma: desde los setentas, los sistemas se masificaron y se diversificaron. Como resultado, se incrementó el número de titulados, pero también la diversidad de títulos o carreras. La inserción al mercado de trabajo de estos egresados también empezó a mostrar vaivenes. Parte de los egresados se queda sin empleo o acepta trabajo en áreas que no corresponden con sus estudios (De Vries y Navarro, 2011; García Montalvo, 2001; Mora et al., 2000). Simultáneamente, las economías pasaron por cambios, con el surgimiento de nuevas actividades económicas en un movimiento hacia la sociedad del conocimiento (Castells, 1997; Planas, 2008). Así, surgió la inquietud sobre el ajuste entre las nuevas demandas de la economía y la oferta correspondiente de la educación superior (Paul et al., 2000).

Ante el aparente desajuste entre la educación superior y el mercado de trabajo, ha nacido un conjunto portentoso de políticas y propuestas de reforma. Un resumen apretado de la literatura revela que la inserción exitosa de egresados al mercado laboral podría depender del tipo de institución, del área de conocimiento, del modelo educativo de la universidad, del contenido curricular, de las formas de enseñanza, de las prácticas profesionales, de las habilidades y competencias adquiridas, del nivel de estudios de los profesores, de las habilidades informáticas y lingüísticas de los egresados, de la movilidad de estudiantes hacia otras instituciones, de las condiciones financieras, del clima organizacional, o del compromiso que siente el estudiante con su institución (Rama, 2011; Barnett y Coate, 2005; Kuh et al., 2005; St. John et al. 2000).

Como resultado, la educación superior ha sido el terreno de un sinnúmero de reformas, ideadas desde el gobierno o desde la misma institución. Un factor común es que todas estas reformas suelen subrayar que, a partir de los cambios realizados, el estudiante saldría mejor preparado para el mercado laboral.

Sin embargo, la relación entre lo que sucede en la universidad y lo que pasa posteriormente en el mercado de trabajo es compleja y sigue siendo poco estudiada (Teichler, 2003). Pascarella y Terenzini (2005) concluyen, a partir de una exhaustiva revisión de la literatura norteamericana, que hay poca evidencia empírica de que la calidad de una institución impacte en el estatus ocupacional de sus egresados. Rumberger y Thomas (1993) señalan que es difícil generalizar sobre los factores institucionales que influyen en las diferencias salariales de los egresados. Igualmente, Hossler y Maple (1993) observan que el nivel educativo de los padres no guarda una relación clara con la carrera educativa y laboral de sus hijos.

\section{Varias contradicciones}

El panorama que surge de la literatura resulta entonces complicado: por un lado, hay un creciente número de estudios sobre la suerte de egresados en el mercado laboral, pero éstos se basan principalmente en el tipo de trabajo y en el ingreso que los egresados perciben. Estos estudios suelen 
enfatizar que los titulados universitarios perciben, en promedio, mejores salarios que aquellos que no lograron terminar una carrera universitaria, pero también que existen desencuentros entre la universidad y el mercado de trabajo. Tales estudios no analizan la experiencia universitaria de los egresados.

Por otra parte, existe una amplia literatura sobre la reorganización y los cambios de la educación superior. Pero estos estudios suelen profetizar sobre las reformas requeridas para que la universidad responda adecuadamente a las nuevas demandas de la economía o del mercado de trabajo. Si bien esta literatura documenta un sinnúmero de reformas y cambios, generalmente no brinda evidencia sobre el impacto de estas reformas en el desempeño de sus egresados en el mercado laboral.

Ambos tipos de investigación raras veces se interconectan. Es decir, los estudios sobre el mercado laboral no analizan factores de preparación o de antecedentes de egresados como posible explicación, mientras que los estudios sobre la universidad y las reformas implementadas no analizan cuáles son los efectos en el mercado laboral. Con ello, queda sin responder una pregunta clave: ¿de qué depende el salario del egresado?

La literatura sugiere tres posibles conjuntos de factores que analizaremos aquí: los antecedentes familiares y educativos; la formación recibida en la universidad, y los factores del mercado de trabajo mismo.

\section{Organización del estudio}

Los datos analizados aquí se basan en un estudio internacional de egresados llamado "El profesional flexible en la sociedad del conocimiento" o PROFLEX. Desde 2006, bajo la coordinación de la Universidad Politécnica de Valencia, PROFLEX ha incorporado a más de 50 instituciones latinoamericanas y se han aplicado más de 10000 cuestionarios. Anteriormente se había aplicado un cuestionario similar en 12 países de la Unión Europea y en Japón (Schomburg y Teichler, 2003), lo cual permite hacer comparaciones entre países.

En el caso mexicano, que analizaremos aquí, participaron nueve universidades: entre las privadas, la Universidad del Valle de México, la Universidad La Salle y CETYS Universidad, e instituciones públicas como el Instituto Tecnológico de Sonora, y las universidades estatales de Guadalajara, San Luís Potosí, Nuevo León, Puebla y Veracruz.

Las nueve universidades aplicaron 4260 cuestionarios a los egresados de licenciatura. Para fines comparativos internacionales, se entrevistaron a personas después de cuatro a cinco años de haber egresado: los cuestionarios fueron aplicados en 2007 y 2008 a aquellos que habían terminado sus estudios en 2002 y 2003. Se trata de un muestreo aleatorio estratificado, proporcional a la población por área geográfica y área de estudio de cada universidad.

La encuesta se llevó a cabo por medio de un cuestionario de preguntas cerradas. La aplicación se efectuó vía entrevistas telefónicas, visitas domiciliarias y a través de una página Web. El margen de error es de $+-0.35 \%$ para los datos globales, considerando $p=q$ y un margen de confianza del 95.5 por ciento.

\section{Limitaciones}

Las universidades mexicanas participantes en PROFLEX representan una parte importante de la matrícula nacional: se trata de universidades públicas y privadas de mediano a gran tamaño, casi todas con más de 50000 estudiantes de licenciatura. No obstante, la muestra también tiene sus limitaciones: en primer lugar, no considera datos sobre algunos sectores del sistema educativo, como las universidades tecnológicas, los institutos tecnológicos, o el amplio sector de pequeñas universidades privadas. En este sentido, los datos 
pueden tener cierto sesgo, ya que solamente se entrevistaron a egresados de universidades consolidadas de reconocido prestigio.

En segundo lugar, la muestra es confiable en el nivel de áreas de conocimiento, pero no en cuanto a las carreras específicas. Un problema importante para estudios de egresados consiste en que casi todas las universidades de tamaño medio o grande actualmente ofrecen más de 50 opciones de licenciatura (a veces hasta 100), algunas con pocos estudiantes y egresados, lo cual complica organizar un estudio representativo para todas las opciones. La agrupación de carreras sigue la acostumbrada en la Unión Europea.

\section{Los ingresos}

La encuesta preguntó a los egresados por su salario mensual bruto. Los datos fueron recalculados en dólares estadounidenses (US\$) en 2008. A partir de los datos reportados, podemos dividir a los egresados en cuatro grupos:

- Grupo 1: menos de US\$ 1000 por mes

- Grupo 2: de US\$ 1001 a US\$ 2000 por mes

- Grupo 3: de US\$2 001 a US\$ 3000 al mes

- Grupo 4: más de US\$ 3000 al mes

La distribución de ingresos se muestra en la tabla 1.

Tabla 1. ¿Cuál es aproximadamente tu sueldo mensual bruto?

Sueldo base regular (US\$ de 2008)

\begin{tabular}{|l|r|r|r|r|}
\hline $\begin{array}{c}\text { Grupo de } \\
\text { ingreso }\end{array}$ & $\begin{array}{c}\text { Media de } \\
\text { ingreso por } \\
\text { grupo (US\$) }\end{array}$ & $\begin{array}{c}\text { \% del total } \\
\text { de ingresos } \\
\text { reportados }\end{array}$ & $\begin{array}{c}\text { \% de la } \\
\text { población que } \\
\text { reporta este } \\
\text { ingreso (N) }\end{array}$ & Población (N) \\
\hline $0-1000$ & 417.76 & 21.2 & 62.4 & 1635 \\
\hline $1001-2000$ & 1446.44 & 30.4 & 25.8 & 194 \\
\hline $2001-3000$ & 2463.15 & 14.9 & 7.4 & 117 \\
\hline Más de 3000 & 9227.26 & 33.6 & & \\
\hline Total & 1227.33 & 100.0 & & \\
\hline
\end{tabular}

Fuente: elaboración propia a partir de datos de PROFLEX. 
E1 $4.5 \%$ de los entrevistados, que reporta ganar más de US\$ 3000 al mes, recibe al 33.6\% del monto salarial total. En cambio, el $62.4 \%$ de la población recibe menos de US\$ 1000 al mes, equivalente al $21.2 \%$ de los ingresos totales. Aunque la distribución pueda parecer extrema, coincide con la distribución general muy desigual de ingresos en México, que reporta un coeficiente GINI de 46.1 (UNDP, 2010).

El promedio de ingreso mensual de nuestra muestra es de US\$ 1227 . En comparación con otros países se trata de salarios bajos: los egresados en Chile reportan en promedio US\$ 1539 . En comparación con la Unión Europea la diferencia es aún más grande: en términos de US\$ ajustados por paridad de poder adquisitivito (PPA), los egresados en México reportan un ingreso de US\$ 1 321, en España 1 632, en Alemania 3210 y en la Gran Bretaña 3222 (Mora et al., 2010).

Analizaremos en adelante los posibles factores que influyen en el ingreso, usando la escala de ingresos de 1 (menos de US\$ 1 000) a 4 (más de US\$ 3000 ) y comparando los cuatro grupos.

\section{La influencia del pasado}

Según la literatura, el capital familiar, expresado en el nivel de escolaridad alcanzado por los padres o los puestos que ocupan, puede jugar un papel importante en el futuro laboral de sus hijos. Los datos confirman esta hipótesis: los egresados con un padre con una escolaridad de escuela primaria reportan, en promedio, un salario de 1.32 (en la escala de 1 a 4), los que tienen un padre con estudios de posgrado un promedio de 1.96. Diferencias semejantes ocurren en cuanto a la escolaridad de la madre. Las diferencias son significativas $(p<0.05)$. El nivel de estudios de los padres es el factor que más parece influir en el éxito laboral del egresado. Mientras mayor sea el nivel educativo de los padres, mayor será el ingreso mensual que obtienen los egresados: en el grupo de menos de 1000 dólares al mes, el 65\% reporta ser de primera generación, es decir, el primer integrante de la familia que accede a la educación superior, en el grupo de US\$ $2001-3000$ es el $41 \%$, en el grupo de más de 3000 es el 33 por ciento.

También se observan diferencias significativas cuando preguntamos por el puesto del padre: cuando el padre ocupa un puesto directivo, el hijo reporta un ingreso de 1.99 , frente a un promedio de 1.50 para puestos intermedios (en una escala de 1 a 4). Aquellos egresados que tienen a sus progenitores en puestos directivos, logran tener ingresos más altos que sus compañeros.

La influencia de la escolaridad y el puesto de los padres hacen suponer que las relaciones que éstos tienen dentro de su campo laboral, ayudan a que los egresados se coloquen en puestos más altos. De esta manera las redes sociales que logran establecer los padres a lo largo de su vida llegan a ser uno de los factores más notorios que determinan la diferencia de sueldos dentro de los cuatro grupos de ingresos que estamos analizando.

Otro factor que influye de manera significante es la experiencia laboral previa al ingreso a la carrera. En el caso de los que ganan más de US\$ 3000 el $32 \%$ reporta haber tenido experiencia laboral relacionada con la carrera, mientras que en el grupo de menos de US\$ 1000 es sólo el 14 por ciento.

El género también tiene un fuerte impacto: los hombres continúan recibiendo un salario más alto que las mujeres. Así, dentro del grupo de menos de 1000 dólares al mes, las mujeres constituyen la mayoría (55\%), mientras en el grupo de 2 a 3 mil representan al \%, y en el grupo de más de 3000 sólo al 24 por ciento.

En cambio, el promedio de calificación obtenido en la educación media superior no tiene mayor impacto. Si bien el grupo de ingresos altos reporta un promedio ligeramente más alto, las diferencias no son significativas $(\mathrm{p}<0.05)$. El hecho que los grupos de ingreso no se distinguen por el promedio 
en la preparatoria causa duda sobre la idoneidad de usar este indicador para el proceso de admisión en las universidades. Existen cuestionamientos sobre el valor predictivo de este factor en cuanto a la culminación exitosa de la carrera (Casillas et al., 2007), pero cuando se trata del éxito en el mercado laboral, el promedio no parece importar.

Sin embargo, también es posible que este factor no incida porque las universidades en la muestra son muy selectivas, requiriendo un promedio de arriba de siete, además de un examen de admisión. Así, la muestra resulta muy homogénea en cuanto a antecedentes académicos. A su vez, un factor ausente en nuestro análisis es el tipo de educación media superior: hay indicios de que las escuelas preparatorias privadas generan resultados distintos que las públicas (De Garay, 2001).

En resumen, en cuanto a los antecedentes, entre los factores que más inciden en el futuro éxito del egresado están la escolaridad de los padres, el puesto de los padres, la experiencia laboral relacionada con el campo de estudio y el género. El promedio de calificación en la educación media superior tiene poca influencia.

\section{La aportación de la universidad (St1)}

¿Hasta dónde contribuye la universidad a las diferencias en ingresos? Una primera comparación es entre las universidades privadas y públicas. En esta comparación se denotan importantes diferencias.

Los egresados de las universidades privadas reportan ingresos más altos que sus colegas de las públicas. Los egresados de la universidad pública reportan un salario de 1.20 , los de la privada 2.03 (en la escala 1 a 4). La diferencia es significativa $(p<0.05)$. Las diferencias se producen incluso dentro de una misma área de conocimiento. También resalta que las mujeres egresadas de la universidad privada suelen ganar salarios más altos que los hombres egresados de la universidad pública, incluso dentro de la misma área de conocimiento.
Lo anterior puede deberse a que los hijos de padres con más escolaridad y más ingresos, tienen mayor acceso económico a las instituciones privadas. Otro factor relacionado es que la mayoría de los jóvenes que egresan de instituciones privadas son de segunda generación en tener acceso a la educación superior. Pero tal como parece, la diferencia en ingresos se debe principalmente al tipo de institución a que se logró acceder.

Un segundo factor es el tipo de carrera o titulación. Si lo analizamos por área de conocimiento, encontramos igualmente diferencias importantes. Las áreas peor remuneradas son Educación (1.17), Ciencias (1.22), Humanidades (1.24), Salud (1.25) y Ciencias Sociales (1.40). Las mejor pagadas son Derecho (1.45), Técnicas (1.70), y Economía y Empresa (1.75). Las últimas dos áreas agrupan las carreras de mayor demanda de acceso y de mayor número de estudiantes inscritos. Dentro de la muestra, el $21.9 \%$ es egresado de Economía y Empresa, y el $28.4 \%$ de Técnicas. En cambio, solamente el 6.5\% es egresado de Humanidades, y el $5.7 \%$ de Ciencias.

Un tercer factor de importancia es la experiencia laboral durante la carrera. En el caso del grupo de menos de US\$1 000 al mes, el 57\% reporta haber tenido experiencia laboral durante la carrera, pero para el grupo de más de US\$ 3000 es el 86 por ciento.

Un cuarto factor son los estudios posteriores: del grupo de ingresos de menos de 1000 dólares al mes, el 33\% reporta haber emprendido estudios de posgrado, frente al \% del grupo con ingresos mayores a 3000 .

Estos datos indicarían que la diferencia en ingresos se explica básicamente por el tipo de universidad y de carrera a que accedió el egresado, por su experiencia laboral o por haber seguido estudios de posgrado. Sin embargo, con estos datos, lo que sucede dentro de la universidad se queda como caja negra. No revelan si las diferencias se 
deben a factores como los métodos de enseñanza, las formas de trabajo, las prácticas profesionales, o el aprendizaje.

\section{El proceso educativo}

Al preguntar a los egresados sobre los métodos de enseñanza, no encontramos diferencias significativas para los cuatro grupos en aspectos como: asistencia a clases, trabajo en grupo, participación en proyectos de investigación, el papel del profesor, las prácticas profesionales, o el énfasis en materias teóricas o prácticas. Los entrevistados reportan las mismas experiencias, sin diferencias entre grupos de ingresos o entre tipos de institución.

La gran similitud en experiencias educativas se expresa con más claridad cuando indagamos acerca de las competencias. El cuestionario PROFLEX pide a los egresados evaluar 19 competencias en tres aspectos: el nivel propio que opina tener el egresado, el nivel exigido en su trabajo actual, y la aportación que hizo la universidad en la adquisición de cada competencia.

En cuanto a las primeras dos dimensiones, encontramos que los de mayores ingresos reportan niveles más altos de competencias propias y de exigencias en el trabajo. Los tres factores que se distinguen mejor entre los cuatro grupos son: capacidad de escribir otros idiomas (correlación de 0.241), capacidad para aplicar conocimientos en la práctica (0.217), y la capacidad de tomar decisiones (0.219). Las demás correlaciones son significativas pero menores a 0.200 .

Esto haría pensar que los grupos de altos ingresos reciben mayores salarios porque alcanzaron niveles más altos de competencias en la universidad. Sin embargo, cuando les preguntamos sobre la aportación que hizo la universidad, las diferencias entre los grupos desaparecen: los cuatro grupos de ingresos evalúan la aportación de su universidad en el mismo nivel. Así, las competencias distintivas parecen haberse adquirido antes de ingresar (como el caso del segundo idioma) o en la práctica laboral (antes, durante o después de los estudios).

Llama la atención que algunos factores guardan una correlación negativa con los salarios recibidos. Uno es el hecho de haber hecho prácticas profesionales relacionadas con la carrera: el 70\% del grupo de menos de 1000 dólares indica haber participado en prácticas, frente al $64 \%$ del grupo que recibe más de US\$ 3000 . Como tal, realizar prácticas profesionales no contribuye a mejores salarios en el futuro. Eso apunta hacia una situación contradictoria: por un lado, la universidad espera que los estudiantes sean de tiempo completo - con becas si tienen bajos ingresos- aun cuando la experiencia laboral durante la carrera resulta tener un efecto positivo posterior en el mercado de trabajo. Por otra parte, las universidades organizan, dentro del currículo, actividades como las prácticas profesionales, donde el estudiante debe pasar unos meses trabajando en una empresa, aun cuando esta actividad resulta no causar mejores ingresos en el futuro. Al parecer, las prácticas profesionales no son consideradas como experiencia laboral.

Algo semejante sucede con la internacionalización. Los que reportan haber hecho una estancia en extranjero durante sus estudios reportan, en promedio, ingresos más bajos.

Los resultados de nuestro análisis de la experiencia formativa son bastante contradictorios frente a las políticas de reforma existentes: por un lado, observamos que existe un número importante de jóvenes que combinan sus estudios con un trabajo $\mathrm{y} / \mathrm{u}$ otra actividad y que por esta razón algunas veces interrumpieron sus estudios. Podría pensarse que quienes ven obstaculizada su trayectoria académica tendrán dificultades para integrarse al campo laboral, pero lo contrario sucede: quienes no tuvieron una interrupción en sus estudios (la mayoría de la población encuestada) 
muestran menores ingresos que quienes sí interrumpieron sus estudios. Nuevamente aparece una contradicción: para tener éxito en el mercado laboral, la experiencia laboral resulta ser un factor más importante que haber sido un estudiante de dedicación de tiempo completo.

Por el otro lado, las experiencias escolarizadas no parecen ser el origen de las diferencias salariales posteriores en el mercado laboral. No hay evidencia que algunos estudiantes salen significativamente mejor preparados, o con distintas competencias, que otros.

\section{El mercado de trabajo}

Finalmente, cabe reparar en los factores del mercado de trabajo mismo. Según nuestros datos, hay diferentes factores que importan para el salario. Un primer factor a tomar en cuenta es la región donde opera la universidad y donde trabaja el egresado: los egresados que estudiaron en el norte de México ganan en promedio menos que los que laboran en el sur. ${ }^{1}$ La diferencia es significativa y confirma que el nivel de ingresos depende de la situación de la economía local.

Un segundo factor es el tipo de trabajo: los que ocupan puestos directivos ganan más al mes que los que ocupan puestos intermedios. Igualmente, los salarios son más altos en el sector privado que en el público. Los egresados que tienen un puesto definitivo ganan más que quienes trabajan con un contrato laboral temporal. También influye el tamaño de la empresa: los que laboran en empresas grandes suelen ganar más que aquellos que están empleados en empresas pequeñas. Finalmente, un factor distintivo, según los entrevistados, es que los puestos mejor remunerados requieren de más años de experiencia laboral para adquirir el nivel de competencias necesarias.
Hay factores que inciden poco: la satisfacción con el trabajo aumenta ligeramente cuando el salario aumenta, pero el incremento no es significativo. Los puestos mejor pagados no requieren niveles más altos de estudio. Finalmente, no hay diferencias significativas entre los cuatro grupos en cuanto a la coincidencia entre estudios y trabajo.

Como tal, la operación cotidiana del mercado de trabajo para egresados universitarios no parece tener mayores secretos: ganan más aquellas personas que ocupan puestos directivos en el sector privado, en empresas grandes, para los cuales se requiere experiencia laboral, con un contrato a largo plazo. Más bien, la interrogante que queda es cómo o por qué algunos egresados pueden acceder a estos puestos y otros no.

\section{Un resumen de los factores que inciden en el salario}

Para indagar cuáles factores impactan más en el nivel salarial de los egresados, realizamos un análisis de regresión lineal, de tres pasos. En el primer paso, consideramos solamente los factores del mercado laboral. En el segundo, incluimos factores de la formación universitaria. En el tercer paso, consideramos los antecedentes familiares. Los resultados en las tablas 2 y 3 .

\section{Discusión de resultados}

La regresión lineal revela varias cosas. En primer lugar, una parte del éxito salarial se puede explicar por las condiciones del mercado de trabajo. Los salarios son más altos en el sur que en el norte. Egresados que logran un puesto directivo tendrán salarios más altos. Los puestos de más altos ingresos requieren más experiencia laboral. El tipo de contrato (indefinido-definido) y el tamaño de la empresa también explican parte de la diferencia.

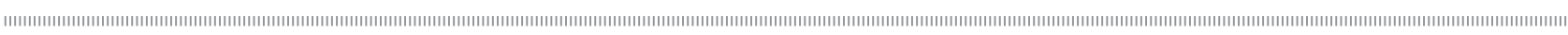
${ }^{1}$ En la muestra, el "Norte" incluye a los estados de San Luis Potosí, Baja California, Jalisco y Nuevo León. El "Sur" incluye al Distrito Federal, Puebla y Veracruz.
} 
Tabla 2. Factores de influencia en el salario de egresados)

\begin{tabular}{|c|c|c|c|c|c|c|c|c|}
\hline \multicolumn{9}{|c|}{ Coeficientes } \\
\hline & \multirow{2}{*}{ Modelo } & \multicolumn{2}{|c|}{$\begin{array}{l}\text { Coeficientes no } \\
\text { estandarizadas }\end{array}$} & \multirow{2}{*}{$\begin{array}{c}\text { Coeficientes } \\
\text { estandarizadas } \\
\text { Beta }\end{array}$} & \multirow{2}{*}{$T$} & \multirow{2}{*}{ Significancia } & \multicolumn{2}{|c|}{$\begin{array}{l}\text { Estadísticas de } \\
\text { colinealidad }\end{array}$} \\
\hline & & $B$ & $\begin{array}{l}\text { Error } \\
\text { estándar }\end{array}$ & & & & Tolerancia & VIF \\
\hline \multirow{6}{*}{$\begin{array}{l}\frac{0}{0} \\
\frac{0}{0} \\
2\end{array}$} & (Constante) & -.037 & .087 & & -.428 & .668 & & \\
\hline & $\begin{array}{l}\text { Región } \\
\text { universidad }\end{array}$ & .388 & .041 & .244 & 9.517 & .000 & .906 & 1.104 \\
\hline & Puesto egresado & .370 & .028 & .342 & 13.248 & .000 & .892 & 1.121 \\
\hline & $\begin{array}{l}\text { Experiencia } \\
\text { requerida }\end{array}$ & .213 & .032 & 165 & 6.747 & .000 & .994 & 1.006 \\
\hline & Tipo contrato & .261 & .049 & .133 & 5.326 & .000 & .959 & 1.043 \\
\hline & $\begin{array}{l}\text { Tamaño empresa } \\
\text { (personal) }\end{array}$ & . 132 & .022 & .148 & 6.042 & .000 & .994 & 1.006 \\
\hline \multirow{6}{*}{$\begin{array}{l}N \\
\frac{0}{0} \\
\frac{0}{0} \\
\sum\end{array}$} & (Constante) & .261 & .081 & & 3.225 & .001 & & \\
\hline & $\begin{array}{l}\text { Región } \\
\text { universidad }\end{array}$ & . 123 & .040 & .077 & 3.075 & .002 & .725 & 1.379 \\
\hline & Puesto egresado & .161 & .027 & .149 & 5.930 & .000 & .727 & 1.375 \\
\hline & $\begin{array}{l}\text { Experiencia } \\
\text { requerida }\end{array}$ & . 188 & .028 & .145 & 6.706 & .000 & .975 & 1.025 \\
\hline & Tipo contrato & .089 & .045 & .045 & 1.993 & .046 & .898 & 1.114 \\
\hline & $\begin{array}{l}\text { Tamaño empresa } \\
\text { (personal) }\end{array}$ & . 044 & .020 & .050 & 2.239 & . 025 & .935 & 1.069 \\
\hline
\end{tabular}




\begin{tabular}{|c|c|c|c|c|c|c|c|c|}
\hline \multirow{3}{*}{$\begin{array}{l}\frac{N}{0} \\
\frac{0}{0} \\
\frac{0}{2}\end{array}$} & $\begin{array}{l}\text { Tipo universidad: } \\
\text { pública-privada }\end{array}$ & .755 & .046 & .466 & 16.387 & .000 & .566 & 1.767 \\
\hline & $\begin{array}{l}\text { Área de } \\
\text { conocimiento }\end{array}$ & .202 & .037 & .124 & 5.517 & .000 & .902 & 1.108 \\
\hline & $\begin{array}{l}\text { Tiene estudios de } \\
\text { posgrado }\end{array}$ & .136 & .036 & .082 & 3.747 & .000 & .949 & 1.054 \\
\hline \multirow{12}{*}{$\begin{array}{l}m \\
\frac{0}{0} \\
\frac{0}{8} \\
\frac{0}{2}\end{array}$} & (Constante) & .053 & .084 & & .634 & .526 & & \\
\hline & $\begin{array}{l}\text { Región } \\
\text { universidad }\end{array}$ & .131 & .039 & .082 & 3.372 & .001 & .724 & 1.381 \\
\hline & Puesto egresado & .134 & .027 & .123 & 5.010 & .000 & .712 & 1.404 \\
\hline & $\begin{array}{l}\text { Experiencia } \\
\text { requerida }\end{array}$ & .176 & .027 & .136 & 6.432 & .000 & .963 & 1.039 \\
\hline & Tipo contrato & .097 & .043 & .049 & 2.234 & .026 & .896 & 1.116 \\
\hline & $\begin{array}{l}\text { Tamaño empresa } \\
\text { (personal) }\end{array}$ & .032 & .019 & .036 & 1.661 & .097 & .925 & 1.081 \\
\hline & $\begin{array}{l}\text { Tipo universidad: } \\
\text { pública-privada }\end{array}$ & .738 & .045 & .456 & 16.454 & .000 & .562 & 1.781 \\
\hline & $\begin{array}{l}\text { Área de } \\
\text { conocimiento }\end{array}$ & .164 & .036 & .101 & 4.557 & .000 & .879 & 1.137 \\
\hline & $\begin{array}{l}\text { Tiene estudios de } \\
\text { posgrado }\end{array}$ & 139 & .035 & .084 & 3.935 & .000 & .947 & 1.056 \\
\hline & Género & .177 & .033 & .113 & 5.287 & .000 & .947 & 1.056 \\
\hline & Puesto padre & .152 & .027 & .122 & 5.720 & .000 & .950 & 1.053 \\
\hline & $\begin{array}{l}\text { Experiencia } \\
\text { laboral antes } \\
\text { de ingresar a la } \\
\text { carrera }\end{array}$ & .172 & .046 & .078 & 3.742 & .000 & .985 & 1.016 \\
\hline
\end{tabular}


Tabla 3. Varianza explicada por cada modelo)

\begin{tabular}{|l|c|c|c|c|c|c|c|c|c|}
\hline Modelo & $R$ & $\begin{array}{c}R \\
\text { Cuadrada }\end{array}$ & $\begin{array}{c}R \\
\text { Cuadrada } \\
\text { Ajustada }\end{array}$ & $\begin{array}{c}\text { Error } \\
\text { Estándar } \\
\text { del } \\
\text { Estimado }\end{array}$ & $\begin{array}{c}\text { Cambio } \\
\text { en } R \\
\text { Cuadrada }\end{array}$ & Cambio F & df1 & df2 & $\begin{array}{c}\text { Significancia } \\
\text { Cambio F }\end{array}$ \\
\hline 1 & $.542 \mathrm{a}$ & .293 & .291 & .65965 & .293 & 98.869 & 5 & 1190 & .000 \\
2 & $.675 \mathrm{~b}$ & .456 & .452 & .57969 & .162 & 117.971 & 3 & 1187 & .000 \\
3 & $.700 \mathrm{c}$ & .490 & .485 & .56205 & .034 & 26.225 & 3 & 1184 & .000 \\
\hline
\end{tabular}

Fuente: elaboración propia a partir de datos de PROFLEX.

a. Predictores: (Constante), Región universidad, Puesto egresado, Experiencia requerida, Tipo contrato, Tamaño empresa (personal)

b. Predictores: (Constante), Región universidad, Puesto egresado, Experiencia requerida, Tipo contrato, Tamaño empresa (personal), Tipo universidad: pública-privada, Área de conocimiento, Tiene estudios de posgrado

c. Predictores: (Constante), Región universidad, Puesto egresado, Experiencia requerida, Tipo contrato, Tamaño empresa (personal), Tipo universidad: pública-privada, Área de conocimiento, Tiene estudios de posgrado, Género, Puesto padre, Experiencia laboral antes de ingresar a la carrera.

Los factores del mercado de trabajo (modelo 1) explican al $29 \%$ de la varianza.

Cuando a la regresión agregamos las posibles aportaciones de la universidad, destaca que los factores significativos son: el tipo de universidad, el tipo de carrera, la experiencia laboral relacionada con la carrera y haber cursado estudios de posgrado. Factores que no influyen son los contenidos curriculares o las competencias adquiridas. El modelo 2 explica al $45 \%$ de la varianza.

Si incluimos a los antecedentes, influyen el género y el puesto del padre y, en menor medida, tener experiencia laboral antes de ingresar a la universidad.

Un aspecto llamativo es que la escolaridad de los padres desvanece como factor estadísticamente significativo cuando lo incorporamos junto a los demás factores en el modelo. Ello no implica que carece de importancia, sino que su impacto depende de la trayectoria del egresado. Como tal, no opera un simple mecanismo de 'origen es destino'. Más bien, para mantener o aumentar su valor, el capital educativo familiar se debe materializar en un conjunto de decisiones acerca del tipo de institución y el tipo de carrera en que se inscribirán los hijos.

En cambio, el puesto del padre sí influye, lo cual indica que la elección de la universidad o la carrera no sólo depende de la escolaridad de los padres, sino de su nivel de ingresos. En este contexto, los padres con mayor escolaridad y mayores ingresos pueden tener mayores posibilidades para colocar a sus hijos en ciertos ámbitos del mercado educativo, por la selectividad y el costo que implican algunas universidades y carreras. Igualmente, pueden tener más acceso a ciertas áreas del mercado laboral, a través de redes sociales. Sin embargo, el camino hacia un puesto de altos ingresos depende en gran medida del estudiante mismo. Hay en este camino múltiples decisiones individuales, aunque en un contexto específico de reglas explícitas e implícitas.

Hay dos factores adicionales de importancia: el género y la experiencia laboral previa al ingreso a la universidad. En el caso de género, las mujeres siguen ganando menores salarios que los hombres, aunque cuenten con la misma preparación. El hecho de que las mujeres suelen obtener un mejor 
promedio de calificación durante la carrera también indica que el desempeño académico es de poca importancia en el mercado laboral. En cambio, la experiencia laboral previa a la carrera sí influye en el éxito. El modelo 3 explica al 49\% de la varianza.

Los datos de colinealidad en la tabla 2 también revelan que los distintos factores son en gran medida independientes entre sí. Ello implica que, por lo menos en teoría, un factor puede compensar a otro. Así, tener experiencia laboral anterior o durante los estudios puede aumentar las posibilidades de obtener un trabajo bien remunerado, no importa el origen social. Igualmente, influye el puesto del padre, aunque pueda carecer de estudios universitarios.

\section{Conclusiones}

Nuestro análisis lleva a algunas conclusiones polémicas. Encontramos que los factores que explican mejor la diferenciación salarial son aspectos del mercado de trabajo mismo, el tipo de institución, el área de conocimiento, la experiencia laboral, el puesto del padre y el género. Es posible que influyan otros factores en el momento de contratación, como la buena presentación, el origen familiar, o el dominio del inglés.

Pero dentro del conjunto de factores, algunos, sorprendentemente, tienen poca relevancia. Un primer factor concierne el capital educativo familiar: ser hijo de padres con estudios universitarios no lleva automáticamente a un futuro laboral con altos ingresos. $\mathrm{Al}$ respecto, el futuro depende mucho más de la universidad y de la carrera elegida.

Un segundo factor de poca relevancia se refiere al currículo o las competencias. Esta ausencia indica que el problema principal no consiste en si la universidad adecuó exitosamente sus planes de estudio a las demandas del mercado laboral.

Encontramos que el factor clave es el tipo de institución: pública o privada de élite. Los egresados de la universidad privada reportan salarios notablemente más altos que sus compañeros de la universidad pública. Sin embargo, no encontramos diferencias significativas entre ambos sectores en términos de formación académica o de calidad. Así, la diferencia parece estar principalmente en el prestigio de la universidad.

Esta diferenciación de prestigios opera por dos vías interconectadas: por un lado, las familias con más capital educativo y financiero, y los jóvenes con mayores aspiraciones salariales, tienden a optar por una universidad privada considerada como de élite. Por el otro lado, en el mercado laboral, los empleadores tienden a reclutar al personal para los puestos mejor pagados desde la universidad privada de élite, lo cual confirma que la decisión del estudiante fue la correcta.

$\mathrm{El}$ ingreso exitoso al mercado, en términos salariales, depende así fuertemente de la imagen de la universidad, tanto para estudiantes como empleadores. Un factor relacionado es el área de conocimiento: destaca que los puestos de altos ingresos se encuentran básicamente en los sectores económico-administrativo (administración, economía) y técnico (ingenierías). En ambas áreas, los sueldos promedios son más altos. Como tal, el éxito en el mercado laboral obedece en gran medida de las estrategias seguidas por los estudiantes y sus padres.

Ahora bien, estas estrategias se desarrollan en un contexto mucho más complejo que en los sesenta, cuando Bourdieu y Passeron (2003) publicaron su obra sobre los "herederos". En aquel entonces, los hijos de la élite social reproducían sus prerrogativas por el sólo hecho de acceder a la universidad y obtener el título. Actualmente, en un contexto de masificación y diversificación de la educación superior, heredar el capital familiar depende fuertemente de la elección adecuada de la carrera y de la institución. Eso implica que las estrategias familiares en muchas ocasiones inician desde la escuela primaria. Significa también 
que los estudiantes - $\mathrm{o}$ sus familias - tengan una noción bien informada acerca de los futuros beneficios de su educación, y que tomen decisiones a partir de esta información (Betts, 1996; Manski, 1993).

Eso también indica que la interacción entre la universidad y el mercado de trabajo es altamente simbólica: los empleadores toman decisiones sobre la contratación de su personal a partir de su impresión de una persona entrevistada y de su universidad de origen. Dentro de este contexto, los pormenores del modelo académico, o los contenidos específicos del currículo, pasarán desapercibidos. Las competencias que cuentan al momento de la contratación son operativas, y difícil de medir de antemano. Además, éstas no se adquieren únicamente en la universidad, sino anteriormente, en el trabajo durante y después de la carrera. Probablemente, la experiencia laboral,

\section{Referencias}

Arrow, K., S. Bowles y S. Durlauf (eds.) (2000), Meritocracy and economic inequality, Princeton, Princeton University Press.

Banco Interamericano de Desarrollo (BID) (2008), Outsiders? The changing patterns of exclusion in Latin America and the Caribbean, Washington, BID.

Barnett, R. y K. Coate (2005), Engaging the curriculum in higher education, Maidenhead, McGraw-Hill.

Becker, G. (1964), Human capital: a theoretical and empirical analysis with special reference to education, Chicago, University of Chicago Press.

Betts, J. R. (1996), "What do students know about wages? Evidence from a survey of undergraduates", en Journal of Human Resources, vol. 31, núm. 1, pp. 27-56.

Brunner, J. J. y D. Uribe (2007), Mercados universitarios. El nuevo escenario de la educación superior, Santiago de Chile, Universidad Diego Portales. más que el título, indica que el egresado ya cuenta con estas competencias.

Lo anterior pone en entredicho el impacto que pueden tener las múltiples reformas universitarias. Cambiar el currículo o introducir cursos especiales (desde inglés hasta cursos de formación de emprendedores) no son acciones que prometen mejorar el éxito de los egresados en el futuro. Como señalan Brunner y Uribe (2007), en la cúspide del sistema universitario, los cambios en el prestigio de una universidad, desde la percepción de sus usuarios, suelen ser marginales.

En este complejo mercado de prestigios e imágenes, quizá habría que reconsiderar la utilidad de las abundantes reformas universitarias, relacionadas con cuantiosas políticas educativas. En cambio, cabría pensar en buenas estrategias de mercadotecnia para mejorar el prestigio de la universidad.
Bolívar, A. (2005), "Equidad educativa y teorías de la justicia", en Revista Iberoamericana sobre Calidad, Eficacia y Cambio en Educación, vol. 3, núm. 2.

Boudon, R. (1983), La desigualdad de oportunidades. La movilidad social en las sociedades industriales, Barcelona, Laia.

Bourdieu, P. y J. C. Passeron (1979), La reproducción. Elementos para una teoría del sistema de enseñanza, Barcelona, Laia.

Bourdieu, P. y J. C. Passeron (2003), Los herederos. Los estudiantes y la cultura, Buenos Aires, Siglo XXI editores.

Bowles, S. y H. Gintis (2000), "Does schooling raise earnings by making people smarter?", en Arrow, K., S. Bowles y S. Durlauf (eds.), Meritocracy and economic inequality, Princeton, Princeton University Press.

Bowles, S. y H. Gintis (2002), "The inheritance of 
inequality", en Journal of Economic Perspectives, 16 (3), pp. 3-30.

Cabrera, A. F., D. J. Weerts y B. F. Zulick (2003), "Encuestas a egresados: tres fundamentos conceptuales en el seguimiento de egresados universitarios", en J. Vidal (coord.), Métodos de análisis de la inserción laboral de los universitarios, Madrid, Ministerio de Educación Cultura y Deporte, Consejo de Coordinación Universitaria, León, Universidad de León, Secretariado de Publicaciones y Medios Audiovisuales.

Casillas, M., R. Chaín y N. Jácome (2007), "Origen social de los estudiantes y trayectorias estudiantiles en la Universidad Veracruzana", en Revista de la Educación Superior, vol. XXXVI (2), núm. 142.

Castells, M. (1997), The information age: economy, society and culture, Cambridge, Blackwell Publishers.

Collins, R. (1979), The credential society: an historical sociology of education and stratification, Nueva York, Academic Press.

De Garay, A. (2001), Los actores desconocidos: una aproximación al conocimiento de los estudiantes, México, Asociación Nacional de Universidades e Instituciones de Educación Superior (ANUIES).

De Vries, W. e Y. Navarro (2011), “¿Profesionistas del futuro o futuros taxistas?" en Revista Iberoamericana de Educación Superior, vol. 2, núm. 4.

Dubet, F. y D. Martuccelli (1998), En la escuela. Sociología de la experiencia escolar, Buenos Aires, Editorial Losada.

García Montalvo, J. (2001), "Educación superior y mercado de trabajo de los titulados universitarios: España frente a Europa", en A. Sáenz de Miera (ed.), En torno al trabajo universitario. Reflexiones y datos, Madrid, Ministerio de Educación, Cultura y Deporte, Consejo de Universidades, pp. 165-194.

Hossler, D. y Maple, S. (1993) "Being undecided about postsecondary education", en Review of Higher Education, 16: 285.

Kuh, G. D., J. Kinzie, J. H. Shuh, E. J. Whitt (2005), Student success in college: creating conditions that matter,
San Francisco, Jossey-Bass.

Manski, C. F. (1993), "Adolescent econometricians: how do youth infer the returns to schooling?", en C. Clotfelter y M. Rothschild (eds.), Studies of supply and demand in higher education, Chicago, National Bureau of Economic Research.

Mora, J. G., J. García-Montalvo y A. García-Aracil (2000), "Higher education and graduate employment in Spain", en European Journal of Education, 35 (2), pp. 229-237.

Mora, J. G., J. M. Carot y A. Conchada (2010), Informe resumen del proyecto PROFLEX en América Latina. Comparativa con el proyecto REFLEX en Europa, Valencia, Universidad Politécnica de Valencia/CEGES.

Pascarella, E. T. y P. T. Terenzini (2005), How college affects students, Volume 2. A third decade of research, San Francisco, Jossey-Bass.

Paul, J. J., U. Teichler y R. van der Velden (eds.) (2000), "Higher education and graduate employment" (special issue), en European Journal of Education, 35 (2).

Pike, G. R. (1994), "The relationship between alumni satisfaction and work experiences", en Research in Higher Education, 35(1), pp. 105-124.

Planas, J. (2008), "El comportamiento de la empleadores mexicanos frente al crecimiento de la educación", en Revista de la Educación Superior, vol. XXXVII (2), núm. 146, pp. 11-40.

Rama, C. (2011), Paradigmas emergentes, competencias profesionales y nuevos modelos universitarios en América Latina, Puebla, Benemérita Universidad Autónoma de Puebla/Educación y Cultura.

Roemer, J. E. (1998), Equality of opportunity, Cambridge, Harvard University Press.

Rumberger, R. W. y S. L. Thomas (1993), "The economic returns to college major, quality and performance: a multilevel analysis of recent graduates", en Economics of Education Review, vol. 12(1), pp. 1-19.

Schomburg, H. y U. Teichler (2003), Higher education and graduate employment in Europe. Results of graduate surveys from 12 countries, Dordrecht, Kluwer Academic Publishers. 
Scott, J. (2005), "Desigualdad de oportunidades y políticas públicas en México: el fracaso del proyecto redistributivo", en SDTE, núm. 330, México, Centro de Investigación y Docencia Económicas.

Sicherman, N. (1991), "'Overeducation' in the labor market", en Journal of Labor Economics, 9 (2), pp. 101-122.

St. John, E. P., A. F. Cabrera, A. Nora y E. H. Asker (2000) "Economic influences on persistence reconsidered. How can finance research inform the reconceptualization of persistence models?", en J. M. Braxton (ed.), Reworking the student departure puzzle: new theory and research on student retention, Nashville, Vanderbilt University Press, pp. 29-47.

Teichler, U. (2001), "Presentación global del Estudio Educación Universitaria y Empleo de los Graduados en Europa. Principales resultados", en A. Sáenz de Miera (ed.), En torno al trabajo universitario. Reflexiones $y$ datos, Madrid, Ministerio de Educación, Cultura y
Deporte, Consejo de Universidades, pp. 123-135.

Teichler, U., (2003) "Aspectos metodológicos de las encuestas a graduados universitarios", en J. Vidal (coord.), Métodos de análisis de la inserción laboral de los universitarios, Madrid, Ministerio de Educación Cultura y Deporte, Consejo de Coordinación Universitaria León, Universidad de León, Secretariado de Publicaciones y Medios Audiovisuales.

United Nations Development Programme (UNDP) (2010), International human development indicators, $<$ http://hdr.undp.org > [consulta: 02 de febrero, 2011].

World Bank (2006), World development report 2006: equity and development, Washington, World Bank.

World Bank (2008), Towards an understanding of socially inherited inequalities in aducational achievement: evidence from Latin America and the OECD, Washington, World Bank, <www.worldbank.org/ lacopportunity $>$ [consulta: 21 de mayo, 2011].

\section{Cómo citar este artículo:}

De Vries, Wietse, Rebeca Vázquez-Cabrera y David Rios-Treto (2013), "Millonarios o malparados: ¿de qué depende el éxito de los egresados universitarios?", en Revista Iberoamericana de Educación Superior (RIES), México, UNAMIISUE/Universia, Vol. IV, núm. 9, pp. 3-20, http://ries.universia.net/index.php/ries/article/view/273/html_37 [consulta: fecha de última consulta]. 